\title{
Quantum critical behavior of itinerant ferromagnets
}

\author{
Thomas Vojta ${ }^{1,2}$, D. Belitz ${ }^{2}$, T. R. Kirkpatrick ${ }^{3}$, and R. Narayanan ${ }^{2}$ \\ ${ }^{1}$ Institut für Physik, Technische Universität Chemnitz, D-09107 Chemnitz, FRG \\ ${ }^{2}$ Dept. of Physics and Materials Science Institute, University of Oregon, Eugene, OR 97403 \\ ${ }^{3}$ Institute for Physical Science and Technology, and Department of Physics, \\ University of Maryland, College Park, MD 20742 \\ vojta@physik.tu-chemnitz.de
}

Received 29 July 1999, accepted dd.mm.yyyy by ue

\begin{abstract}
We investigate the quantum phase transition of itinerant ferromagnets. It is shown that correlation effects in the underlying itinerant electron system lead to singularities in the order parameter field theory that result in an effective long-range interaction between the spin fluctuations. This interaction turns out to be generically antiferromagnetic for clean systems. In disordered systems analogous correlation effects lead to even stronger singularities. The resulting long-range interaction is, however, generically ferromagnetic.

We discuss two possibilities for the ferromagnetic quantum phase transition. In clean systems, the transition is generically of first order, as is experimentally observed in MnSi. However, under certain conditions the transition may be continuous with non-mean field critical behavior. In disordered systems, one finds a very rich phase diagram showing first order and continuous phase transitions and several multicritical points.
\end{abstract}

Keywords: quantum phase transitions, quantum magnets, critical behavior

PACS: $75.20 . \mathrm{En} ; 75.45 .+\mathrm{j} ; 64.60 . \mathrm{Kw}$

\section{Introduction}

Quantum phase transitions are phase transitions that occur at zero temperature as a function of some non-thermal control parameter. The fluctuations that drive these transition are of quantum nature rather than thermal in origin. Among the transitions that have been investigated are various metal-insulator transitions, the superconductor-insulator transition in thin metal films, and a variety of magnetic phase transitions. Quantum phase transitions have attracted considerable attention in recent years, in particular since they are believed to be at the heart of some of the most exciting discoveries in modern condensed matter physics, such as the localization problem, various magnetic phenomena, the quantum Hall effects, and hightemperature superconductivity [1].

One of the most obvious examples of a quantum phase transition is the transition from a paramagnetic to a ferromagnetic metal that occurs as a function of the exchange coupling between the electron spins. The experimentally best studied example of such a transition is probably provided by the pressure-tuned transition in $\mathrm{MnSi}$ [2]. $\mathrm{MnSi}$ belongs to the class of so-called nearly or weakly ferromagnetic materials. This group of metals, consisting of transition metals and their compounds such as $\mathrm{ZrZn}_{2}, \mathrm{TiBe}_{2}$, 


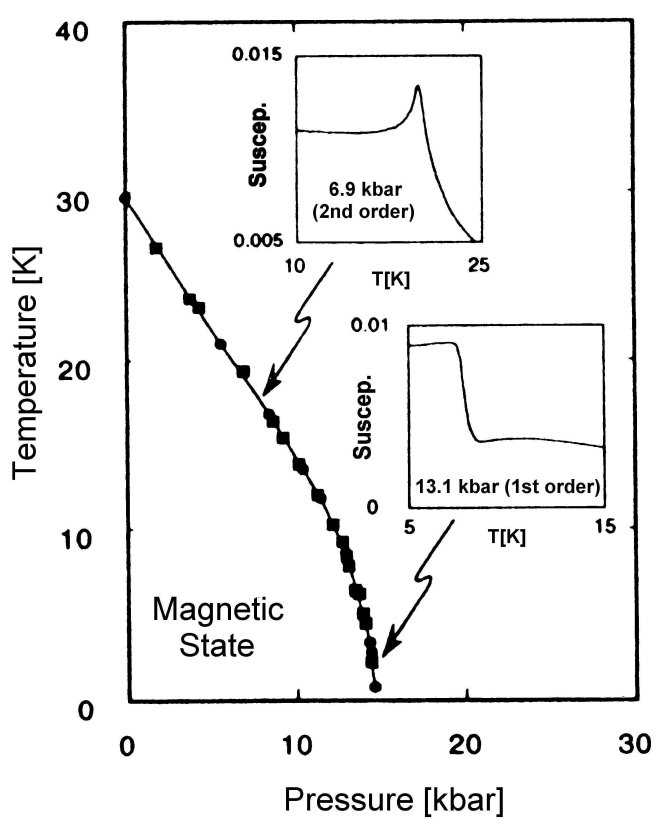

Fig. 1 Phase diagram of MnSi. The insets show the behavior of the susceptibility close to the transition. (After [2]).

$\mathrm{Ni}_{3} \mathrm{Al}$, and $\mathrm{YCo}_{2}$ in addition to $\mathrm{MnSi}$ are characterized by strongly enhanced spin fluctuations. Thus, their ground state is close to a ferromagnetic instability which makes them good candidates for actually reaching the ferromagnetic quantum phase transition experimentally by changing the chemical composition or applying pressure.

At ambient pressure MnSi is paramagnetic for temperatures larger than $T_{c}=30 \mathrm{~K}$. Below $T_{c}$ it orders magnetically. The order is, however, not exactly ferromagnetic but a long-wavelength $(190 \AA)$ helical spin spiral along the (111) direction of the crystal. The ordering wavelength depends only weakly on the temperature, but a homogeneous magnetic field of about $0.6 \mathrm{~T}$ suppresses the spiral and leads to ferromagnetic order. One of the most remarkable findings about the magnetic phase transition in $\mathrm{MnSi}$ is that it changes from continuous to first order with decreasing temperature as is shown in Fig. 1. Specifically, in an experiment carried out at low pressure (corresponding to a comparatively high transition temperature) the susceptibility shows a pronounced maximum at the transition, reminiscent of the singularity expected from a continuous phase transition. In contrast, in an experiment at a pressure very close to (but still smaller than) the critical pressure the susceptibility does not show any sign of a divergence at the phase transition. Instead, it displays a finite discontinuity suggestive of a first-order phase transition.

A related set of experiments is devoted to a phenomenon called itinerant electron metamagnetism. Here a high magnetic field is applied to a nearly ferromagnetic material such as $\mathrm{Co}\left(\mathrm{Se}_{1-x} \mathrm{~S}_{x}\right)_{2}$ or $\mathrm{Y}\left(\mathrm{Co}_{1-x} \mathrm{Al}_{x}\right)_{2}$ [3]. At a certain field strength the 
magnetization of the sample shows a pronounced jump. This can easily be explained if we assume that the free energy as a function of the magnetization has the triplewell structure characteristic of the vicinity of a first-order phase transition. In zero field the side minima must have a larger free energy than the center minimum (since the material is paramagnetic in zero field). The magnetic field essentially just "tilts" the free energy function. If one of the side minima becomes lower than the center (paramagnetic) one, the magnetization jumps.

In the literature the first-order transition in $\mathrm{MnSi}$ at low temperatures as well as the itinerant electron metamagnetism have been attributed to sharp structures in the electronic density of states close to the Fermi energy which stem from the band structure of the particular material. These structures in the density of states can lead to a negative quartic coefficient in a magnetic Landau theory and thus to the above mentioned triple-well structure.

In this paper it will be shown, however, that the two phenomena are generic since they are rooted in the universal many-body physics underlying the transition. Therefore, they are predicted to occur for all nearly or weakly ferromagnetic materials irrespective of special structures in the density of states. In this paper we will emphasize the basic physics behind these findings, a technical derivation can be found elsewhere [4, 6, 6, 7].

The paper is organized as follows: In Sec. 2 we sketch the derivation of an order parameter field theory for the ferromagnetic quantum phase transition of itinerant electrons, starting from a microscopic description of an interacting electron system and analyze its properties. In Sec. 3 we compare the possible scenarios for the quantum phase transition in detail, while Sec. $⿴$ tis devoted to the influence of quenched disorder. We conclude in Sec. 5 .

\section{Order parameter field theory}

In a pioneering paper that was the first application of the modern theory of critical phenomena to a quantum phase transition, Hertz [8] derived an order parameter field theory for the ferromagnetic quantum phase transition by considering a simple model of itinerant electrons that interact only via the exchange interaction in the particlehole spin-triplet channel. Hertz analyzed this order parameter field theory by means of renormalization group (RG) methods. He found a continuous phase transition whose critical behavior in the physical dimensions $d=3$ and $d=2$ is mean field-like, since the dynamical critical exponent $z=3$ decreases the upper critical dimension from $d_{c}^{+}=4$ for the classical case to $d_{c}^{+}=1$ in the quantum case. Despite the somewhat artificial nature of this model, it was believed for a long time that the qualitative features of Hertz's analysis, in particular the fact that there is mean field-like critical behavior for all $d>1$, apply to real itinerant quantum ferromagnets as well.

Here we will show, however, that this belief is mistaken. The properties of the ferromagnetic quantum phase transition are much more complicated since the magnetization couples to additional, non-critical soft modes in the electronic system. Mathematically, this renders the conventional Landau-Ginzburg-Wilson approach invalid since an expansion of the free energy in powers of the order parameter does not ex- 
ist. Physically, the additional soft modes lead to an effective long-range interaction between the order parameter fluctuations. This long-range interaction, in turn, can change the character of the transition from a continuous transition with mean-field exponents to either a continuous transition with non-trivial (non-mean field) critical behavior or even to a first order transition like in $\mathrm{MnSi}$.

The derivation of our theory [4] follows Hertz [8] in spirit, but the technical details are considerably different. We consider a $d$-dimensional continuum model of interacting electrons, and pay particular attention to the particle-hole spin-triplet contribution [9] to the interaction term in the action, $S_{\text {int }}^{t}$, whose (repulsive) coupling constant we denote by $\Gamma_{t}$. Writing only the latter explicitly, and denoting the spin density by $\mathbf{n}_{s}$, the action reads,

$$
S=S_{0}+S_{\text {int }}^{t}=S_{0}+\left(\Gamma_{t} / 2\right) \int d x \mathbf{n}_{s}(x) \cdot \mathbf{n}_{s}(x),
$$

where $S_{0}$ contains all contributions to the action other than $S_{\text {int }}^{t}$. In particular, it contains the particle-hole spin-singlet and particle-particle interactions, which will be important for what follows. $\iint d x=\int d \mathbf{x} \int_{0}^{1 / T} d \tau$, and we use a 4-vector notation $x=$ $(\mathbf{x}, \tau)$, with $\mathbf{x}$ a vector in real space, and $\tau$ imaginary time. Following Hertz, we perform a Hubbard-Stratonovich decoupling of $S_{\mathrm{int}}^{t}$ by introducing a classical vector field $\mathbf{M}(x)$ with components $M^{i}$ that couples to $\mathbf{n}_{s}(x)$ and whose average is proportional to the magnetization, and we integrate out all fermionic degrees of freedom. We obtain the partition function $Z$ in the form

$$
Z=e^{-F_{0} / T} \int D[\mathbf{M}] \exp [-\Phi[\mathbf{M}]],
$$

where $F_{0}$ is the non-critical part of the free energy. The Landau-Ginzburg-Wilson (LGW) functional $\Phi$ reads

$$
\begin{aligned}
\Phi[\mathbf{M}]= & \frac{1}{2} \int d x d y \frac{1}{\Gamma_{t}} \delta(x-y) \mathbf{M}(x) \cdot \mathbf{M}(y) \\
& +\sum_{n=2}^{\infty} a_{n} \int d x_{1} \ldots d x_{n} \chi_{i_{1} \ldots i_{n}}^{(n)}\left(x_{1}, \ldots, x_{n}\right) M^{i_{1}}\left(x_{1}\right) \ldots M^{i_{n}}\left(x_{n}\right),
\end{aligned}
$$

where $a_{n}=(-1)^{n+1} / n$ !. The coefficients $\chi^{(n)}$ in (3) are connected n-point spin density correlation functions of a reference system with action $S_{0}$ [5, 8]. The particle-hole spin-triplet interaction $\Gamma_{t}$ is missing in the bare reference system, but a nonzero $\Gamma_{t}$ is generated perturbatively by the particle-particle interaction contained in $S_{0}$. The reference system then has all of the characteristics of the full action $S$, except that it must not undergo a phase transition lest the separation of modes that is implicit in our singling out $S_{\text {int }}^{t}$ for the decoupling procedure breaks down.

$\chi^{(2)}$ is the spin susceptibility of the reference system. Performing a Fourier transform from $x=(\mathbf{x}, \tau)$ to $q=(\mathbf{q}, \Omega)$ with wave vector $\mathbf{q}$ and Matsubara frequency $\Omega$, we have for small $\mathbf{q}$ and $\Omega$,

$$
\chi^{(2)}(\mathbf{q}, \Omega)=\chi_{0}(\mathbf{q})[1-|\Omega| /|\mathbf{q}|],
$$

${ }^{a}$ We note, however, that even in Hertz's original model, where $S_{0}$ describes free electrons, interaction terms get generated upon renormalization. The traditional mean-field results therefore are not correct for this model either. 
where $\mathbf{q}$ and $\Omega$ are being measured in suitable units, and $\chi_{0}(\mathbf{q})$ is the static spin susceptibility of the reference system. If we take the susceptibility to be that of a non-interacting Fermi gas, $\chi_{0}(\mathbf{q} \rightarrow 0)=c_{0}-c_{2} \mathbf{q}^{2}$, we obtain Hertz's theory. However, in a real Fermi liquid, the static spin susceptibility at $T=0$ is a non-analytic function of $\mathbf{q}$ since the magnetization modes couple to additional soft modes, viz. particle-hole excitations in the spin-triplet channel with a ballistic dispersion relation [6]. This nonanalyticity is crucial for the non-trivial physics of the itinerant ferromagnets discussed in this paper. For small wave vectors the static susceptibility is of the form

$$
\chi_{0}(\mathbf{q} \rightarrow 0)=c_{0}-c_{d-1}|\mathbf{q}|^{d-1}-c_{2} \mathbf{q}^{2} .
$$

Here $c_{0}, c_{d-1}$ and $c_{2}$ are constants. This holds for $1<d<3$; in $d=3$ the nonanalyticity is of the form $-\tilde{c}_{2} \mathbf{q}^{2} \ln |1 / \mathbf{q}|$. Note that all these singularities only exist at zero temperature and in zero magnetic field since both a finite temperature or a magnetic field gives the particle-hole excitations a mass.

Using (四可), and with $\int_{q}=\sum_{\mathbf{q}} T \sum_{i \Omega}$, the Gaussian part of $\Phi$ can be written,

$$
\Phi^{(2)}[\mathbf{M}]=\int_{q} \mathbf{M}(q)\left[t_{0}+c_{d-1}|\mathbf{q}|^{d-1}+c_{2} \mathbf{q}^{2}+c_{\Omega}|\Omega| /|\mathbf{q}|\right] \mathbf{M}(-q) .
$$

Here $t_{0}=1-\Gamma_{t} \chi^{(2)}\left(\mathbf{q} \rightarrow 0, \omega_{n}=0\right)$ is the bare distance from the critical point, and $c_{\Omega}$ is another constant.

For the same physical reasons for which the non-analyticity occurs in (5), the higher coefficients $\chi^{(n)}(n>2)$ in (3) are in general not finite at zero frequencies and wave numbers. Generally, the coefficient of $|\mathbf{M}|^{n}$ in $\Phi$ for $|\mathbf{p}| \rightarrow 0$ behaves like $\chi^{(n)}=v^{(n)}|\mathbf{p}|^{d+1-n}$. This implies that $\Phi$ contains a non-analyticity which in our expansion takes the form of a power series in $|\mathbf{M}|^{2} /|\mathbf{p}|^{2}$. Consequently, the free energy functional (3) is mathematically ill defined. However, we will nonetheless be able to extract a considerable amount of information.

The sign of the non-analyticity in the Gaussian term merits some attention since it will be responsible for the qualitative features of the ferromagnetic quantum phase transition. Perturbation theory to second order in $\Gamma_{t}$ yields $c_{d-1}<0$ [四, 6]. This is the generic case, and it is consistent with the well-known notion that correlation effects in general decrease the effective Stoner coupling [10]. However, Ref. [6] has given some possible mechanisms for $c_{d-1}$ to be positive at least in some materials.

\section{Phase transition scenarios}

Depending on the sign of the non-analyticity in the Gaussian term (6) of the free energy functional the properties of the ferromagnetic quantum phase transition will be qualitatively different.

We first discuss the generic case of $c_{d-1}<0$. Here the free energy reduces with increasing $q$ from zero which implies that a continuous transition to a ferromagnetic state is impossible at zero temperature. Two possible scenarios for the phase transition arise for $c_{d-1}<0$. The first scenario is based on the observation that a finite thermodynamic magnetization $m=\langle|\mathbf{M}(x)|\rangle$, which acts similarly to a magnetic field, 
cuts off the singularities in the coefficients of the order parameter field theory. Therefore, the non-analyticity in $\chi^{(2)}$ leads to an analogous non-analyticity in the magnetic equation of state, which takes the form

$$
\begin{array}{rll}
t m-v_{d} m^{d}+u m^{3} & =H & (d<3), \\
t m-v_{3} m^{3} \ln (1 / m)+u m^{3} & =H & (d=3),
\end{array}
$$

where $t$ tunes the transition and $u, v_{d}$ and $v_{3}$ are positive constants. $H$ denotes the external magnetic field. This equation of state describes a first-order phase transition since the next-to-leading term for small $m$ has a negative sign. We have investigated this scenario in some detail [7]. Since the non-analyticities in $\chi^{(2)}$ and the equation of state are cut off by a finite temperature, the transition will be of first order at very low $T$ but turn second order at higher temperatures. The two regimes are separated by a tricritical point.

The second possible scenario for the quantum phase transition arising if $c_{d-1}<0$ is that the ground state of the system will not be ferromagnetic but instead a spindensity wave at finite q. This scenario has not been studied in much detail so far, but work is in progress. It is tempting to interpret the spiral ordering in $\mathrm{MnSi}$ as a signature of this finite- $q$ instability. This is, however, not very likely since a finite- $q$ instability caused by our long-range interaction will be strongly temperature dependent due to the temperature cutoff of the singularities. As mentioned above, experimentally the ordering wave vector is essentially temperature independent. Further work will be necessary to decide which of the two possible scenarios, viz. a first-order ferromagnetic transition or a continuous transition to modulated magnetic order, is realized under what conditions. Moreover, let us point out, that in $d=3$ the non-analyticity is only a logarithmic correction and would hence manifest itself only as a phase transition at exponentially small temperatures, and exponentially large length scales. Thus, it may well be unobservable experimentally for some materials.

We now turn to the second case, $c_{d-1}>0$ which can happen, if one of the conditions discussed in Ref. [6] is fulfilled. In this case the self-generated long-range interaction is ferromagnetic. Consequently, the ferromagnetic quantum phase transition will be a conventional second order phase transition, which can be analyzed by standard renormalization group methods. A tree level analysis shows that the Gaussian theory is sufficient for dimensions $d>d_{c}^{+}=1$ since all higher order terms are irrelevant. We are therefore able to obtain the critical behavior exactly, yet due to the long-range interaction it is not mean field-like. The results of this analysis [4] can be summarized as follows. The equation of state close to the critical point reads

$$
\begin{array}{rll}
t m+v_{d} m^{d}+u m^{3} & =H & (d<3), \\
t m+v_{3} m^{3} \ln (1 / m)+u m^{3} & =H & (d=3),
\end{array}
$$

Again, $u$ and $v$ are positive constants. Note the different sign of the non-analytic term compared to (8). From (9,10) one obtains the critical exponents $\beta$ and $\delta$, defined by $m \sim t^{\beta}$ and $m \sim H^{1 / \delta}$, respectively, at $T=0$. For $\beta$ and $\delta$, and for the correlation length exponent $\nu$, the order parameter susceptibility exponent $\eta$, and the dynamical exponent $z$, we find

$$
\beta=\nu=1 /(d-1), \quad \eta=3-d, \quad \delta=z=d \quad, \quad(1<d<3),
$$


and $\beta=\nu=1 / 2, \eta=0, \delta=z=3$ for $d>3$. These exponents 'lock into' mean-field values at $d=3$, but have nontrivial values for $d<3$. In $d=3$, there are logarithmic corrections to power-law scaling. Eqs. (9, 10) apply to $T=0$. At finite temperature, we find homogeneity laws for $m$, and for the magnetic susceptibility, $\chi_{m}$,

$$
\begin{aligned}
& m(t, T, H)=b^{-\beta / \nu} m\left(t b^{1 / \nu}, T b^{\phi / \nu}, H b^{\delta \beta / \nu}\right), \\
& \chi_{m}(t, T, H)=b^{\gamma / \nu} \chi_{m}\left(t b^{1 / \nu}, T b^{\phi / \nu}, H b^{\delta \beta / \nu}\right),
\end{aligned}
$$

where $b$ is an arbitrary scale factor. The exponent $\gamma$, defined by $\chi_{m} \sim t^{-\gamma}$ at $T=$ $H=0$ and the crossover exponent $\phi$ that describes the crossover from the quantum to the classical Heisenberg fixed point (FP) are given by

$$
\gamma=\beta(\delta-1)=1 \quad, \quad \phi=\nu,
$$

for all $d>1$. Notice that the temperature dependence of the magnetization is not given by the dynamical exponent. However, $z$ controls the temperature dependence of the specific heat coefficient, $\gamma_{V}=c_{V} / T$, which has a scale dimension of zero for all $d$, and logarithmic corrections to scaling for all $d<3$ [11,

$$
\gamma_{V}(t, T, H)=\Theta(3-d) \ln b+\gamma_{V}\left(t b^{1 / \nu}, T b^{z}, H b^{\delta \beta / \nu}\right) .
$$

Eqs. (9) - (15) represent the exact critical behavior of itinerant quantum Heisenberg ferromagnets for all $d>1$ with the exception of $d=3$, where additional logarithmic corrections to scaling appear 偖.

\section{Influence of disorder}

In this section we briefly discuss the influence of quenched non-magnetic disorder on the ferromagnetic quantum phase transition. An approach along the lines of the one for the clean case sketched in Sec. 2 has been developed in Ref. [5], and the resulting effective theory is very similar. Again, the magnetization couples to additional soft modes (here with diffusive dynamics) which leads to an effective long-range interaction. The singularities are even stronger than in the clean case, but they have the opposite sign so that the long-range interaction is generically ferromagnetic. Thus in the presence of disorder there will be a competition between the ballistic and diffusive singularities. and the temperature which cuts off both. For weak disorder the first-order transition will survive, while larger disorder leads to a continuous transition. As shown in Ref. [7], the phase diagram becomes very rich, showing several multicritical points and even regions with metamagnetic behavior (see Fig. 2). The properties of the continuous quantum phase transition occurring for stronger disorder can again be analyzed by

standard renormalization group methods. It turns out that as in the clean case the Gaussian theory is sufficient since all higher order terms are irrelevant. The resulting critical exponents are

$$
\gamma=1,
$$

for all $d>2$,

$$
\nu=1 /(d-2) \quad, \quad \eta=4-d \quad, \quad z=d,
$$




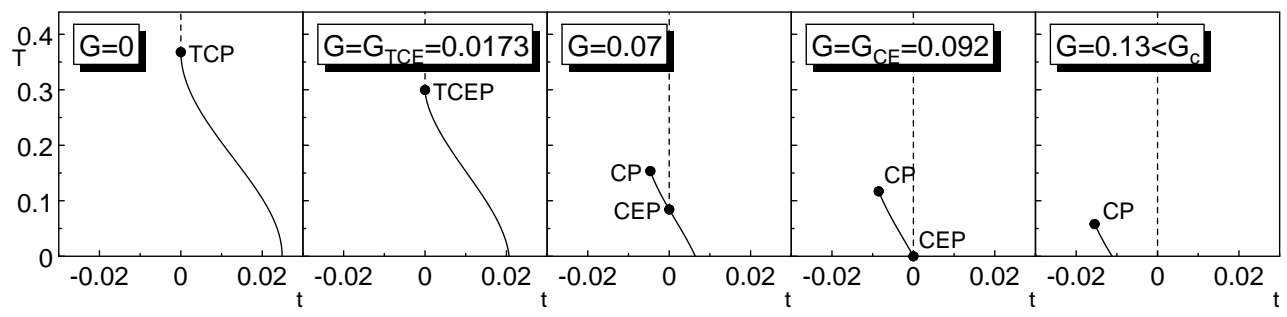

Fig. 2 Phase diagrams of disordered itinerant ferromagnets in the $T$ - $t$-plane showing first order (solid) and second order (dashed) transitions. $G$ is a dimensionless measure of the disorder, and CP, CEP, TCP, and TCEP denote critical points, critical endpoints, tricritical points and tricritical endpoints, respectively. See Ref. [7] for more details.

for $2<d<4$, while $\nu=1 / 2, \eta=0$, and $z=4$ for $d>4$. In addition to $d=4, d=6$ also plays the role of an upper critical dimension, and one has

$$
\beta=2 /(d-2) \quad, \quad \delta=d / 2,
$$

for $2<d<6$, while $\beta=1 / 2, \delta=3$ for $d>6$.

An important problem in disordered systems that has attracted a lot of attention within the last years is the influence of rare disorder fluctuations on the properties of the phase transition. In the conventional perturbative approach [5] the rare regions are neglected. We have developed a theory which includes the rare regions into a renormalization group approach. This theory is discussed in a separate paper in this volume 13. Here we quote only the final result for the itinerant ferromagnet: Due to the effective long-range interaction which stabilizes the Gaussian theory, rare regions do not change the properties of the ferromagnetic quantum phase transition (in contrast to, e.g., itinerant antiferromagnets).

\section{Conclusions}

To summarize, we have discussed the ferromagnetic quantum phase transition in itinerant electron systems. It has been shown that the critical magnetization modes couple to additional, non-critical soft modes in the electronic systems which results in an effective long-range interaction between the magnetization fluctuations. We have discussed several possible scenarios for the ferromagnetic quantum phase transition. In clean systems the generic scenario is a first-order phase transition. This provides us with a complete explanation for the nature of the transitions observed in $\mathrm{MnSi}$, which in Ref. 20 were attributed to a band structure feature characteristic of $\mathrm{MnSi}$. While this feature may well be sufficient to make the transition in $\mathrm{MnSi}$ of first order, the present theory leads to the surprising prediction that the first order transition is generic, and thus should be present in other weak clean itinerant ferromagnets as well. Our theory further predicts in detail how the first order transition will be suppressed by quenched disorder. Observations of such a suppression, or lack thereof, would be 
very interesting for corroborating or refuting the theory. Semi-quantitatively, the theory predicts that the $T$ region that shows a first order transition will be largest for strongly correlated systems. Conversely, since the dependence of the tricritical temperature on the system parameters is exponential, in some, or even many, systems the first order transition may take place only at very low temperatures. This may explain why in $\mathrm{ZrZn}_{2}$ no first order transition has been observed [2], although the experiment does not seem to rule out a weakly first order transition [12].

Under certain conditions, the transition in clean itinerant ferromagnets can also be of second order. These conditions have been formulated mathematically. Their precise physical or experimental nature has not been investigated so far, but it is known that strong correlations are a necessary condition. If these conditions are fulfilled, then the critical behavior is known exactly, albeit it is not mean field-like. If the system contains sufficiently strong quenched disorder, the transition is always of second order, and the non-mean field-like critical behavior has been determined exactly.

Finally, it has been shown that the presence of rare regions or local moments in the disordered case does not change these results, in contrast to, e.g., the case of itinerant antiferromagnets. The reason lies in the effective long-range interaction between the order parameter fluctuations in the ferromagnetic case, which is sufficient to suppress all fluctuations at the critical point, including the static disorder fluctuations responsible for rare regions. The same suppression of fluctuations is also the reason behind our ability to determine the critical behavior for bulk systems, and indeed for any dimension, exactly.

We thank G. Lonzarich and C. Pfleiderer for helpful discussions, and the Aspen Center for Physics for hospitality during the completion of this paper. This work was supported by the NSF under grant Nos. DMR-98-70597 and DMR-99-75259, and by the DFG (SFB 393/C2).

\section{References}

[1] See, e.g., S. Sachdev, A.V. Chubukov, and A. Sokol, Phys. Rev. B 51 (1995) 14874; S.L. Sondhi, S.M. Girvin, J.P. Carini, and D. Shahar, Rev. Mod. Phys. 69 (1997) 315: S.-C. Zhang, Science 275 (1997) 1089; T.R. Kirkpatrick and D. Belitz, cond-mat/9707001, to appear in Electron Correlations in the Solid State, edited by N.H. March, Imperial College Press

[2] C. Pfleiderer, G.J. McMullan, S.R. Julian, and G.G. Lonzarich, Phys. Rev. B 55 (1997) 8330, and references therein

[3] K. Adachi et al., J. Phys. Soc. Japan 47 (1979) 675; T. Sakakibara, T. Goto, K. Yoshimura, and K. Fukamichi J. Phys. Condens. Matter 2 (1990) 3381

[4] T. Vojta, D. Belitz, R. Narayanan, and T.R. Kirkpatrick, Europhys. Lett. 36 (1996), 191; Z. Phys. B 103 (1997) 451

[5] T.R. Kirkpatrick and D. Belitz, Phys. Rev. B 53 (1996) 14364

[6] D. Belitz, T.R. Kirkpatrick, and T. Vojta, Phys. Rev. B 55 (1997) 9452

[7] D. Belitz, T.R. Kirkpatrick and T. Vojta, Phys. Rev. Lett 82 (1999) 4707

[8] J. Hertz, Phys. Rev. B 14 (1976) 1165

[9] See, e.g., A. A. Abrikosov, L. P. Gorkov, and I. E. Dzyaloshinski, Methods of Quantum Field Theory in Statistical Physics, Dover, New York 1975, Sec.5

[10] R.M. White, Quantum Theory of Magnetism, McGraw-Hill, New York 1970

[11] F. J. Wegner, Phys. Rev. B 5 (1972) 4529 has shown how 'resonance' conditions between critical exponents lead to logarithmic corrections to scaling. Their appearance for a whole range of dimensionalities in $(15)$ is a consequence of the exact relation $z=d$ 
[12] C. Pfleiderer, private communication

[13] R. Narayanan, Thomas Vojta, D. Belitz, and T.R. Kirkpatrick, Ann. Phys. (Leipzig) 8 (1999) SI-185-8 